\title{
AN EFFICIENT LEGENDRE PSEUDOSPECTRAL METHOD FOR SOLVING NONLINEAR QUASI BANG-BANG OPTIMAL CONTROL PROBLEMS
}

\author{
EMRAN TOHIDI AND SOMAYYEH LOTFI NOGHABI
}

\begin{abstract}
In this paper, an efficient Legendre pseudospectral approach for the accurate solution of nonlinear quasi bang-bang optimal control problems (OCPs) is investigated. In this approach, after linearizing the dynamical system, control and state functions are considered as piecewise constant and piecewise continuous polynomials, respectively, and the switching points are also taken as decision variables. Furthermore, for simplicity in discretization, a integral formulation of the dynamical equations is considered. Thereby, the problem is converted into a mathematical programming problem which can be solved by well-developed parameter optimization algorithms. Through a numerical implementation we show the efficiency of the proposed method via comparing with a classical pseudospectral method and other discretization approaches.
\end{abstract}

Mathematics Subject Classification 2000: 65N35, 49J15, 90C30, 93C05, 49M25, 90C05. Additional Key Words and Phrases: Quasi Bang-Bang optimal control; Switching point; Numerical methods; Pseudospectral Legendre method

\section{INTRODUCTION}

Optimal control problems (OCPs), which are also called trajectory optimization problems, appear in a wide class of applications. Apart from aerospace engineering, robotics and chemical engineering, optimal control theory has also been used with great success in economics and biomedicine (see [3] and [4]) .

In the real world, and because of the limitations in energy and other sources, controllers are always restricted. Therefore, it is need to impose bounds for the control function. This assumptions often leads to discontinuous controls (see [20]). As a first practical implementation of Legendre polynomials for solving discontinuous OCPs, one can refer to a well-known paper that was published recently by Shamsi [20]. In the mentioned paper, the author use the modified Legendre approximation for solving discontinuous bang-bang OCPs. Moreover, the theoretical analysis (including the existence and convergence of optimal solutions) related to the discontinuous OCPs, can be found in [12]. In this paper, by using the general idea of [20] we solve nonlinear quasi bang-bang optimal control problems numerically. In other words, we linearize the dynamical system (in the main nonlinear problem), and then we use the idea of Shamsi for dealing with the approximated

DOI 10.2478/v10294-012-0016-0

CUniversity of SS. Cyril and Methodius in Trnava 


\section{EMRAN TOHIDI, SOMAYYEH LOTFI}

OCP, which is a bang-bang OCP, and since the considered problems in this paper can be reduced to bang-bang OCPs we call these problems as quasi bang-bang OCPs.

We recall that, there exist two main class of methods for solving OCPs; the first is optimizing then discretizing and the second is discretizing then optimizing. The first class can be named as indirect approaches and the second class can be named as direct techniques. Although the Hamiltonian-Jacobi-Bellman (HJB) equations provide a framework for obtainig the optimal solutions, they suffer from well-known fundamental problems [2], such as the nonsmoothness of the value function and the "curse of dimensionality". The alternative framework of the Minimum Principle, while more tracable from a control-theoretic point of view, generates open loop controls if it can be solved at all. The Minimum Principle approach is also beset with fundamental numerical problems due to the fact that the costates are adjoint to the state perturbation equations [3]. In other words, the Hamiltonian generates a numerically sensitive boundary value problem that may produce such wild trajectories as to exceed the numerical range of the computer [5]. To overcome this difficulty, direct methods have been employed to solve complex OCPs arising in many applied fields [3].

The pseudospectral methods are powerful tools for numerical solution of ordinary and partial differential equations, which can be traced back to 1970s (see [6] and [11]). If we want to solve an ordinary or partial differential equation to high accuracy on a simple domain, and if the data defining the problem are smooth, then the pseudospectral methods are usually the best tool. They can often achieve 10 digits of accuracy where a finite difference scheme or a finite element method would get two or three [25]. At lower accuracies, they demand less computational time and computer memory than the alternatives. For a recent efficient use of pseudospectral methods, in physical and engineering problems, see [6], [7], [8], [9], [16], [18] and [19].

The pseudospectral method has been successfully applied in solving smooth OCPs [8]. In this method, polynomial approximations of the state and control variables are considered where Lagrange polynomials are the trial functions and the unknown coefficients are the values of the state and control variables at the Legendre-GaussLobatto (LGL) or Chebyshev- Gauss-Lobatto (CGL) points. It is well known that this choice of collocation points yields superior results for interpolation of functions to the ones obtained from equidistant points [11]. By using the properties of the Lagrange polynomials, the state equations and the control constraints are readily transformed to algebraic equations. The state differential constraints are imposed by evaluating the functions at the LGL points and using a derivative matrix that is obtained by taking the analytic derivative of the interpolating polynomials and evaluating them at the LGL points. The integral cost function can also be discretized by Gauss-Lobatto quadrature rules, which provide highly accurate results for approximating integrals [6]. The OCP is thereby converted into a nonlinear programming problem (NLP), which can be solved by a well-developed parameter optimization algorithm [10], [14]. However, in this paper the MATLAB optimization routine FMINCON is used for solving the resulting NLP.

There are highly efficient direct optimization approaches for solving OCPs such 


\section{AN EFFICIENT LEGENDRE PSEUDOSPECTRAL METHOD}

as [3], [4] and [24]. The pseudospectral methods are one of them that are efficient and accurate for solving smooth OCPs [3]. In contrast, using the pseudospectral methods in solving bang-bang OCPs can cause several issues and high-order accuracy of method is deteriorated. Because bang-bang optimal control problems have discontinuities in the control and in the derivatives of the states. In this case, the pseudospectral methods exhibit the well-known Gibbs phenomenon [11] resulting from the approximation of a nonsmooth function by a finite number of smooth functions. Also, switching points in control can not be captured by these methods and adding more nodes, for overcoming these difficulties, could lead to defficiencies and ill-conditioning of the discretized problem. The following example depicts the defficiency of the classical pseudospectral methods for solving discontinuous OCPs.

Example 1.1. [17] Consider the following bang-bang OCP

$$
\begin{array}{ll}
\min & J=\frac{1}{2} \int_{0}^{5}\left(x_{1}^{2}(\tau)+x_{2}^{2}(\tau)\right) d \tau \\
\text { s.t. } & \dot{x}_{1}(\tau)=x_{2}(\tau), \\
& \dot{x}_{2}(\tau)=-x_{1}(\tau)+x_{2}(\tau)+u(\tau), \quad 0 \leq \tau \leq 5, \\
& x_{1}(0)=0.231, \quad x_{2}(0)=1.126, \\
& |u(\tau)| \leq 0.8, \quad 0 \leq \tau \leq 5 .
\end{array}
$$

The computational interval can be changed from $[0,5]$ into $[-1,1]$ by a simple transformation as done in the following OCP

$$
\begin{array}{ll}
\min & J=\frac{5}{4} \int_{-1}^{1}\left(x_{1}^{2}(t)+x_{2}^{2}(t)\right) d t \\
\text { s.t. } & \dot{x}_{1}(t)=\frac{5}{2} x_{2}(t), \\
& \dot{x}_{2}(t)=\frac{5}{2}\left(-x_{1}(t)+x_{2}(t)+u(t)\right), \quad-1 \leq t \leq 1, \\
& x_{1}(-1)=0.231, \quad x_{2}(-1)=1.126, \\
& |u(t)| \leq 0.8, \quad-1 \leq t \leq 1 .
\end{array}
$$

The optimal control of the above-mentioned problem is given as follows [26]

$$
u^{*}(t)=\left\{\begin{array}{cc}
-0.8 & -1 \leq t \leq-0.49, \\
0.8 & -0.49 \leq t \leq 1
\end{array}\right.
$$

If one apply a classic pseudospectral method like as [13] for solving this problem, then the desired optimal control can not be obtained in a suitable way. The best obtained numerical solution with $N=32$ is depicted in Figure 1. From this Figure one can see that not only the exact value of switching point (i.e., $t=-0.49$ ) is not detected with a high accuracy (since the obtained switching point has 0.1 error), but also the obtained solution has additional jumps in the boundary of domain. These are the disadvantages of the applying the traditional pseudospectral methods for solving nonsmooth problems.

To overcome all the mentioned numerical difficulties, a modified pseudospectral scheme is presented in [20] for solving bang-bang OCPs that it has two major 
EMRAN TOHIDI, SOMAYYEH LOTFI

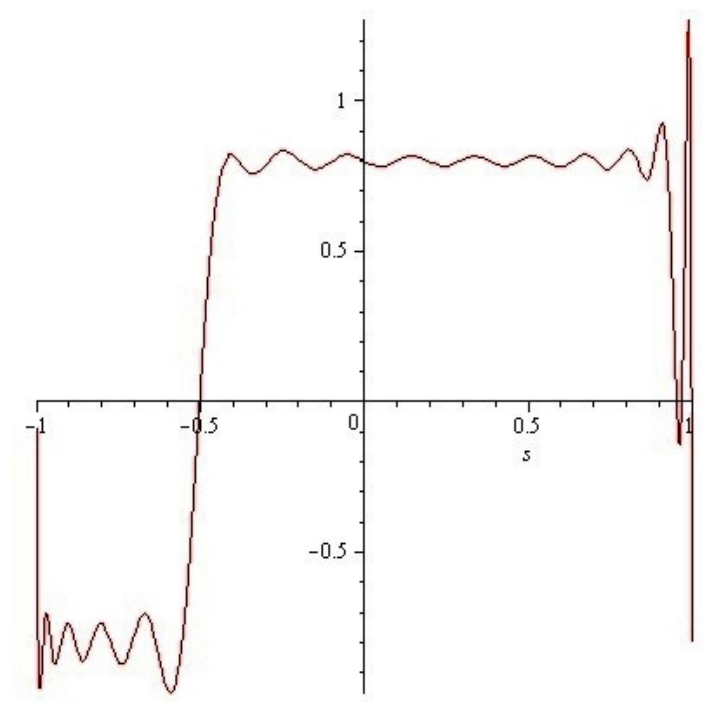

Fig. 1. The best numerical solution for the control function of example 1.1. for $N=32$

differences with the traditional pseudospectral methods. First, instead of approximating the states and controls by a polynomial in the whole computational domain, as suggested in the classical pseudospectral methods, the author of [20] looks for a piecewise continuous approximation polynomial for each state and a piecewise constant function for each control function. For this purpose, the computational domain is partitioned into subdomains where the size of each subdomain is considered as an unknown parameter and this enables us to compute the switching times. Second, in contrast with the traditional pseudospectral methods, the integral form of the OCP is discretized. This equivalent form is found by integrating the differential dynamics and adding in the initial conditions. The advantage of using the integral form lies in the fact that this form releases us of imposing the patching conditions for continuity enforcement of states in the boundary of subdomains.

We mention that both pseudospectral methods and method of [20] belong to direct methods for numerical solution of OCPs. Direct methods have been used extensively in a variety of trajectory optimization problems [4]. Their advantage over indirect methods, which rely on solving the necessary conditions derived from Pontryagins minimum principle, is their wider radius of convergence to an optimal solution, therefore they do not need a good initial guess. In addition, since the necessary conditions need not be derived, the direct methods can be quickly used to solve a number of practical trajectory optimization problems.

In this paper, we use the technique of [20] for solving a class of nonlinear OCPs that can be converted into bang-bang OCPs, which are called quasi bang-bang OCPs, after linearizing the dynamical system according to the given suitable conditions on controls. Quasi bang-bang OCPs are considered in several papers by Tohidi and his coworkers (see [15], [22] and [23]). In [15] and [23] the authors use the simplest form of finite difference for dealing with these problems (i.e. quasi 


\section{AN EFFICIENT LEGENDRE PSEUDOSPECTRAL METHOD}

bang-bang OCPs). With more details for discretizing the dynamical system they use the forward and backward (of order one) techniques for approximating the derivatives and for approximating the integral that involved in the cost function they use the trapozeidal scheme. Moreover the technique of [22], which is similar to [13], is suggested for these problems. However, several difficulties like as there exist in Example 1.1. can be apear in dealing with these problems.

\section{PROBLEM STATEMENT AND LINEARIZATION OF THE DYNAMICAL SYS- TEM}

\subsection{Problem Statement}

Consider the following nonlinear (in terms of control) dynamical system

$$
\dot{x}(t)=A(t) x(t)+H(t, u(t))
$$

with known initial and final conditions $x(0)=x_{0}, x\left(t_{f}\right)=x_{f}$, where $x(t)$ and $u(t)$ are $n \times 1$ and $q \times 1$ state and control vectors respectively, $A(t) \in \Re^{n \times n}$ is a continuous matrix function and $u(t) \in U$ where $U$ is a compact and connected subset of $\Re^{q}$. For clarity of presentation, it is assumed that $n=q$ and $H:\left[0, t_{f}\right] \times U \longrightarrow \Re^{n}$ is a continuous function over $\left[0, t_{f}\right] \times U$. Moreover, there exists a pair of state and control variables $(x(t), u(t))$ such that satisfies (1) and two point boundary conditions $x(0)=x_{0}$ and $x\left(t_{f}\right)=x_{f}$ together with the assupmtion $u(t) \in U$. The problem is to find the optimal control $u(t)$ and the corresponding state trajectory $x(t), 0 \leq t \leq t_{f}$, satisfying Eq. (1) while minimizing the cost functional

$$
J=\int_{0}^{t_{f}} f(t, x(t)) d t .
$$

\subsection{Linearization of the dynamical system}

Since $H:\left[0, t_{f}\right] \times U \longrightarrow \Re^{n}$ is a continuous function and $\left[0, t_{f}\right] \times U$ is a compact and connected subset of $\Re^{n+1}$, then $\{H(t, u(t)): u \in U\}$ is a closed set in $\Re^{n}$ clearly. Thus, $\left\{H_{i}(t, u(t)): u \in U\right\}$ for $i=1,2, \ldots, n$ is closed in $\Re$. Now, suppose that the lower and upper bounds of the $\left\{H_{i}(t, u(t)): u \in U\right\}$ are $g_{i}(t)$ and $w_{i}(t)$ respectively. Therefore,

$$
g_{i}(t) \leq H_{i}(t, u(t)) \leq w_{i}(t) \quad t \in\left[0, t_{f}\right] .
$$

In other words

$$
\begin{array}{ll}
g_{i}(t)=\min _{u}\left\{H_{i}(t, u(t)): u \in U\right\} & t \in\left[0, t_{f}\right], \\
w_{i}(t)=\max _{u}\left\{H_{i}(t, u(t)): u \in U\right\} & t \in\left[0, t_{f}\right] .
\end{array}
$$

Using linear combination property of intervals, which is clarified in [22], $H_{i}(t, u(t))$ can be expressed as a convex linear combination of its minimum $g_{i}(t)$ and maximum $w_{i}(t)$ as follows

$$
H_{i}(t, u(t))=\lambda_{i}(t) w_{i}(t)+\left(1-\lambda_{i}(t)\right) g_{i}(t)=\lambda_{i}(t) \beta_{i}(t)+g_{i}(t),
$$

where $\beta_{i}(t)=w_{i}(t)-g_{i}(t)$ and $\lambda_{i}(t) \in[0,1]$.

We recall that, $\lambda_{i}(t)$ is the new associated control variable. 
Now, the main problem is transformed into the following OCP

$$
\begin{array}{ll}
\min & \int_{0}^{t_{f}} f(t, x(t)) d t \\
\text { S.t. } & \dot{x}(t)=A(t) x(t)+B(t) \Lambda(t)+g(t), \\
& \Lambda(t) \in \overbrace{[0,1] \times[0,1] \times \ldots \times[0,1]}^{n \text { times }}, \quad t \in\left[0, t_{f}\right], \\
& x(0)=x_{0}, \quad x\left(t_{f}\right)=x_{f},
\end{array}
$$

where $B(t)=\operatorname{diag}\left(\beta_{1}(t), \beta_{2}(t), \cdots, \beta_{n}(t)\right)_{n \times n}, \Lambda(t)=\left(\lambda_{1}(t), \lambda_{2}(t), \cdots, \lambda_{n}(t)\right)_{n \times 1}$ and $\left.g(t)=\left(g_{1}(t), g_{2}(t), \cdots, g_{n}(t)\right)\right)_{n \times 1}$. Note that problem $(7)$ is a bang-bang OCP, because in this problem the new control $\Lambda(t)$ has lower 0 and upper 1 bounds and appears linearly in the dynamical equations. As soon as the controls are assumed to be bang-bang, the problem of finding the required controls becomes one of finding the switching times.

Corollary 2.1. Let the pair of $\left(x^{*}(),. \Lambda^{*}().\right)$ be the optimal solution of problem (7). Then, there exists $u^{*}($.$) such that the pair of \left(x^{*}(),. u^{*}().\right)$ is the optimal solution of problem (1)-(2).

Proof. Let $u^{*}($.$) satisfies systems of (6), where \Lambda^{*}($.$) is replaced by \Lambda($.$) . Thus, the$ pair of $\left(x^{*}(),. u^{*}().\right)$ satisfies condition (1). Since the objective function of problem (1)-(2) is the same of problem (7), then the pair of $\left(x^{*}(),. u^{*}().\right)$ is the optimal solution of (1)-(2) evidently.

\section{BASIC IDEA: DISCRETIZATION}

We consider that $n=1$ (i.e., $\Lambda(t)=\lambda(t)$ ) and assume that optimal bang-bang problem (7) has a solution with $s \geq 1$ switching points denoted by $t_{1}, t_{2}, \cdots, t_{s}$. So if we set $t_{0}=0$ and $t_{s+1}=t_{f}$, then the total domain $\left[0, t_{f}\right]$ breaks into $s+1$ subdomains. i.e.

$$
\left[0, t_{f}\right]=\left[t_{0}, t_{1}\right] \cup\left[t_{1}, t_{2}\right] \cup \ldots \cup\left[t_{s}, t_{s+1}\right],
$$

where on each subdomain, $\lambda(t)$ is constant. We denote $\lambda(t)$ in $k$ th subdomain with constant vector $b^{k}$. Since $0 \leq \lambda(t) \leq 1$, we have

$$
0 \leq b^{k} \leq 1, \quad k=1,2, \ldots, s+1 .
$$

Also we denote the restriction of $x(t)$ to the $k$ th subdomain by $x^{k}(t)$. Note that we use superscript $k$ in $x^{k}(t)$ and $b^{k}$ to signify the $k$ th subdomain.

Given the decomposition (8), the dynamic equations of (7) are expressed as

$$
\begin{aligned}
& \dot{x}^{k}(t)=A(t) x^{k}(t)+B(t) b^{k}+g(t), \quad t_{k-1} \leq t \leq t_{k}, \quad k=1,2, \ldots, s+1 \\
& x^{1}(0)=x_{0} \\
& x^{k}\left(t_{k-1}\right)=x^{k-1}\left(t_{k-1}\right), \quad k=2,3, \ldots, s+1 .
\end{aligned}
$$

Note that Equation (11) is considered to guarantee the continuity of state functions.

We now integrate Equation (9) to conclude the following integral form of dynamic equations

$$
x^{k}(t)=c^{k-1}+\int_{t_{k-1}}^{t}\left(A(\tau) x^{k}(\tau)+B(\tau) b^{k}+g(\tau)\right) d \tau, k=1, \ldots, s+1,
$$




\section{AN EFFICIENT LEGENDRE PSEUDOSPECTRAL METHOD}

where $t_{k-1} \leq t \leq t_{k}$ and

$$
c^{k}=\left\{\begin{array}{rc}
x_{0} & k=0 \\
x^{k}\left(t_{k}\right) & k=1,2, \ldots, s
\end{array}\right.
$$

We use the above equations instead of Equations (9)-(11) in the discretization procedure.

The terminal conditions $x\left(t_{f}\right)=x_{f}$ are applied only on $x^{s+1}(t)$ as

$$
x^{s+1}\left(t_{s+1}\right)=x_{f} .
$$

Herewith, problem (7) is converted to the following multidomain minimization problem:

$$
\begin{array}{ll}
\min & J=\sum_{k=1}^{s+1} \int_{t_{k-1}}^{t_{k}} f\left(\tau, x^{k}(\tau)\right) d \tau \\
\text { s.t. } & x^{k}(t)=c^{k-1}+\int_{t_{k-1}}^{t}\left(A(\tau) x^{k}(\tau)+B(\tau) b^{k}+g(\tau)\right) d \tau \\
& t_{k-1} \leq t \leq t_{k}, \quad k=1,2, \cdots, s+1 \\
& x^{s+1}\left(t_{s+1}\right)=x_{f} \\
& 0 \leq b^{k} \leq 1, \quad k=1,2, \cdots, s+1 .
\end{array}
$$

The above multidomain form is more convenient than the original problem (7) for discretization by the pseudospectral methods. The advantages of using the multidomain form lie in the fact that, first, the switching points are considered as parameters in problem and this enables switching time values to be computed readily and precisely. Second, with our assumption $x^{k}(t)$ is smooth on $\left(t_{k-1}, t_{k}\right)$ and so we can expand it in Lagrange bases with spectral accuracy in each subdomain.

For discretizing the problem (15), let $\hat{\tau}_{i}^{k}, i=0,1, \ldots, N$, be the shifted LGL nodes to subdomain $\left[t_{k-1}, t_{k}\right]$, i.e. $\hat{\tau}_{i}^{k}=\left(\tau_{i}\right) \frac{t_{k}-t_{k-1}}{2}+\frac{t_{k}+t_{k-1}}{2}$. By using Lagrange interpolation, we approximate $x^{k}(t)$ by

$$
x^{k}(t) \approx \sum_{i=0}^{n} a_{i}^{k} \hat{L}_{i}^{k}(t)
$$

where $a_{i}^{k}$ is an $n$-vector such that $a_{i}^{k}=x^{k}\left(\hat{\tau}_{i}^{k}\right)$ and

$$
\hat{L}_{i}^{k}(t)=L_{i}\left(\frac{2}{t_{k}-t_{k-1}} t-\frac{t_{k}+t_{k-1}}{t_{k}-t_{k-1}}\right)
$$

We recall that $L_{i}(t)$ is the $i$ th Lagrange base which is constructed from LGL nodes $\tau_{i}$ for all $i=0,1, \ldots, N$.

Using (16), we can approximate $f$ in $k$ th subdomain as

$$
\begin{aligned}
f\left(\tau, x^{k}(\tau)\right) & \approx \sum_{j=0}^{N} f\left(\hat{\tau}_{j}^{k}, x^{k}\left(\hat{\tau}_{j}^{k}\right)\right) \hat{L}_{j}^{k}(t) \\
& =\sum_{j=0}^{N} f\left(\hat{\tau}_{j}^{k}, a_{j}^{k}\right) \hat{L}_{j}^{k}(t) .
\end{aligned}
$$


EMRAN TOHIDI, SOMAYYEH LOTFI

Now by substituting approximations (16) and (17) into (15), we get

$\sum_{j=0}^{N} a_{j}^{k} \hat{L}_{j}^{k}(t)=c^{k-1}+\int_{t_{k-1}}^{t}\left(A(\tau) \sum_{j=0}^{N} a_{j}^{k} \hat{L}_{j}^{k}(\tau)+B(\tau) b^{k}+g(\tau)\right) d \tau \quad t_{k-1} \leq t \leq t_{k}$,

From (13) and (16) for $k=1, \ldots, s$, we have $c^{k}=a_{N}^{k}$. Now if we set $a_{N}^{0}=x_{0}$, then we obtain

$$
c^{k}=a_{N}^{k}, \quad k=0, \ldots, s+1 .
$$

By collocating equation (18) in $\hat{\tau}_{i}^{k}, i=0, \ldots, N, k=1, \cdots, s+1$, we have (note that in (18) we consider a fix $k$ and then collocating (18) $N+1$ times for the considered $k$ )

$$
\begin{aligned}
a_{i}^{k} & =a_{N}^{k-1}+\sum_{j=0}^{N} a_{j}^{k} \int_{t_{k-1}}^{\hat{\tau}_{i}^{k}} A(\tau) \hat{L}_{j}^{k}(\tau) d \tau+b^{k} \int_{t_{k-1}}^{\hat{\tau}_{i}^{k}} B(\tau) d \tau+\int_{t_{k-1}}^{\hat{\tau}_{i}^{k}} g(\tau) d \tau, \quad i=0,1, \ldots, N \\
& =a_{N}^{k-1}+\frac{t_{k}-t_{k-1}}{2}\left(\sum_{j=0}^{N} a_{j}^{k} \int_{-1}^{\tau_{i}} A(\tau) L_{j}(\tau) d \tau+b^{k} \int_{-1}^{\tau_{i}} B(\tau) d \tau+\int_{-1}^{\tau_{i}} g(\tau) d \tau\right)
\end{aligned}
$$

For approximating the integrals that involved in the above equations we need to Legendre Gauss Lobatto quadrature rule. For this reason, by applying simple linear transformations, we first change the interval $\left[-1, \tau_{i}\right]$ into $[-1,1]$ as follows

$$
\tau=\frac{\tau_{i}+1}{2} \eta+\frac{\tau_{i}-1}{2}, \quad i=0,1, \ldots, N .
$$

Thus we can apply Legendre Gauss Lobatto rule as follows

$$
\begin{aligned}
a_{i}^{k} & =a_{N}^{k-1}+\frac{\left(t_{k}-t_{k-1}\right)}{2} \frac{\left(\tau_{i}+1\right)}{2}\left(\sum_{j=0}^{N} a_{j}^{k} \int_{-1}^{1} A(\eta) L_{j}(\eta) d \eta+b^{k} \int_{-1}^{1} B(\eta) d \eta+\int_{-1}^{1} g(\eta) d \eta\right) \\
& \approx a_{N}^{k-1}+\frac{\left(t_{k}-t_{k-1}\right)}{2} \frac{\left(\tau_{i}+1\right)}{2}\left\{\sum_{l=0}^{N} w_{l}\left(\sum_{j=0}^{N} a_{j}^{k} A\left(\tau_{l}\right) L_{j}\left(\tau_{l}\right)+b^{k} B\left(\tau_{l}\right)+g\left(\tau_{l}\right)\right)\right\}, 0 \leq i \leq N,
\end{aligned}
$$

where $w_{l}=\frac{2}{N(N+1)} \frac{1}{P_{N}^{2}\left(\tau_{l}\right)}$ for $l=0,1, \ldots, N$ is the LGL weights and $P_{N}(x)$ is the $N$ th degree Legendre Polynomial.

So the by noting that $x^{s+1}\left(t_{f}\right)=a_{N}^{s+1}$, problem (15) is discretized to the following NLP:

$$
\begin{array}{ll}
\min & J_{N, s}=\sum_{k=1}^{s+1} \sum_{j=0}^{N} \sum_{l=0}^{N} \frac{t_{k}-t_{k-1}}{2} f\left(\hat{\tau}_{j}^{k}, a_{j}^{k}\right) w_{l} L_{j}\left(\tau_{l}\right) \\
\text { s.t. } & a_{i}^{k}-a_{N}^{k-1}+\frac{\left(t_{k}-t_{k-1}\right)}{2} \frac{\left(\tau_{i}+1\right)}{2}\left\{\sum_{l=0}^{N} w_{l}\left(\sum_{j=0}^{N} a_{j}^{k} A\left(\tau_{l}\right) L_{j}\left(\tau_{l}\right)+b^{k} B\left(\tau_{l}\right)+g\left(\tau_{l}\right)\right)\right\}=0 \\
& i=0,1, \ldots, N, \quad k=1,2, \cdots, s+1 \\
& a_{N}^{s+1}-x_{f}=0, \quad \\
& 0 \leq b^{k} \leq 1, \quad k=1,2, \cdots, s+1
\end{array}
$$




\section{AN EFFICIENT LEGENDRE PSEUDOSPECTRAL METHOD}

Here, the vectors $b^{k}, a_{i}^{k}, i=0, \cdots, N, k=1, \cdots, s$ and parameters $t_{1}, \cdots, t_{s}, t_{f}$ are unknown variables in the NLP. Note that $a_{N}^{0}$ is known and $a_{N}^{0}=x_{0}$.

In the above discretization procedure, the number of switching points, $s$, is considered as a known parameter. So at first we must guess the number of switching points. However, as illustrated in [20], the method is capable of finding the correct number of switching points even if the procedure starts with an incorrect guess of the number of switching points. Note that, if the number of switching points, $s$, is chosen correctly, then the resulting value of $b^{k}$ is equal to its lower or upper bounds, furthermore $b^{k}$ changes in each switching point.

\section{NUMERICAL ILLUSTRATION}

In this section, we apply our scheme for solving a typical nonlinear quasi bang-bang OCP. This example was derived first in [15] and then provided in [22] and [23]. As a first practical technique, which is based on the simpleset form of finite difference approximations, in [15] we solve the following example numerically and comparisons with another approach [1] confirm the applicability of the method of [15]. One of the advantages of the method of [15] is that we finally solve a Linear Programming (LP) problem. However, this method have other disadvantages such as needing to higher values of approximations (i.e., $N$ ) and this leads to ill-conditioning of the approximated problem. Since then, in [22] we apply Legendre (polynomial) approximations for dealing with nonlinear quasi bang-bang OCPs so that the obtained results in [22] are superior with respect to a classic collocation method [21]. However this spectral approach do not requires huge number of collocation nodes, but since the optimal control is discontinuous several difficulties (that illustrated in Example 1.1.) can be appear in this problems. If we apply the presented method for solving this problem, we have both advantages of the methods of [15] and [22]. The proposed method do not contains the above difficulties of the classic spectral methods for solving nonsmooth OCPs and also achieve superior results with respect to at least 4 other methods. These advantages confirm the efficiency of this modern spectral approximation. The following example is modeled using the mathematical software package MATLAB and the corresponding nonlinear programming problem is solved using the command FMINCON. All calculations are run on a Pentium 4 PC Laptop with $2 \mathrm{GHz}$ of CPU and 2 GB RAM.

Example 4.1. Consider the following nonlinear quasi bang-bang OCP

$$
\begin{array}{ll}
\min & J=\int_{0}^{1}(|\sin (2 \pi t)|-\exp (-t)) x(t) d t \\
\text { s.t. } & \dot{x}(t)=\left(t^{5}-t^{2}+t\right) x(t)-|u(t)|^{3} \exp (\sin (2 \pi t)), \\
& u(t) \in[-1,1], \quad x(0)=0.9, \quad x(1)=0.4
\end{array}
$$

We recall that, this problem is a nonlinear OCP. According to the condition on control, this OCP can be reduced to a linear OCP which is generally is a bangbang OCP with the new associated control $\lambda(t)$.

In this problem we have $H(t, u(t))=-|u(t)|^{3} \exp (\sin (2 \pi t))$, and then

$$
\begin{aligned}
& g(t)=\min _{u}\{H(t, u(t)): u \in[-1,1]\}=-\exp (\sin (2 \pi t)) \\
& w(t)=\max _{u}\{H(t, u(t)): u \in[-1,1]\}=0 .
\end{aligned}
$$


EMRAN TOHIDI, SOMAYYEH LOTFI

\begin{tabular}{cccccc} 
& Table I. & \multicolumn{4}{c}{ Numerical results of Example 4.1. } \\
\hline$N$ & $t_{1}$ & $t_{2}$ & $t_{3}$ & $t_{4}$ & $J_{N}$ \\
\hline 6 & 0.0891 & 0.1984 & 0.4137 & 0.7385 & -0.0526 \\
8 & 0.0800 & 0.2171 & 0.4597 & 0.7089 & -0.0500 \\
9 & 0.0800 & 0.2064 & 0.4248 & 0.7128 & -0.0511 \\
10 & 0.0800 & 0.2093 & 0.4421 & 0.7081 & -0.0505 \\
11 & 0.0800 & 0.2150 & 0.4538 & 0.7051 & -0.0500 \\
12 & 0.0800 & 0.2189 & 0.4623 & 0.7024 & -0.0496 \\
13 & 0.0800 & 0.2090 & 0.4408 & 0.7041 & -0.0503 \\
14 & 0.0800 & 0.2136 & 0.4502 & 0.7030 & -0.0500 \\
15 & 0.0800 & 0.2171 & 0.4577 & 0.7010 & -0.0497 \\
16 & 0.0800 & 0.2088 & 0.4398 & 0.7020 & -0.0502 \\
\hline
\end{tabular}

Table II. Comparisons of the Methods in evaluating the objective function $J^{*}$

\begin{tabular}{cccccc}
\hline$N$ & proposed method & method of $[22]$ & method of $[21]$ & method of $[15]$ & method of $[1]$ \\
\hline 6 & -0.0526 & -0.0331 & -0.0354 & - & - \\
8 & -0.0500 & -0.0346 & -0.0356 & - & - \\
10 & -0.0505 & -0.0370 & -0.0368 & - & - \\
11 & -0.0500 & -0.0387 & -0.0377 & - & - \\
100 & - & - & - & -0.0434 & -0.0261 \\
\hline
\end{tabular}

Therefore $B(t)=w(t)-g(t)=\exp (\sin (2 \pi t))$ and hence $H(t, u(t))$ can be approximated as follows

$$
\begin{aligned}
H(t, u(t)) & \approx B(t) \lambda(t)+g(t) \\
& \approx \exp (\sin (2 \pi t)) \lambda(t)-\exp (\sin (2 \pi t)) .
\end{aligned}
$$

Considering this approximation, the main problem can be approximated with the following bang-bang OCP

$$
\begin{array}{ll}
\min & J=\int_{0}^{1}(|\sin (2 \pi t)|-\exp (-t)) x(t) d t \\
\text { s.t. } & \dot{x}(t)=\left(t^{5}-t^{2}+t\right) x(t)+\exp (\sin (2 \pi t)) \lambda(t)-\exp (\sin (2 \pi t)), \\
& \lambda(t) \in[0,1], \quad x(0)=0.9, \quad x(1)=0.4
\end{array}
$$

For exhibiting further discussions we assume that the numer of switching points is $s=4$. Since by applying this assumption we reach to the exact results in which the new associated control $\lambda(t)$ is switched between its lower and upper bound. The numerical results related to the values of switching points and objective function for different values of $N$ are adopted in Table 1. Moreover the optimal state $x(t)$, associated control $\lambda(t)$ and control $u(t)$ are depicted in Figures 2, 3 and 4. Moreover, in Table 2 comparisons of the numerical results of the proposed method with respect to the methods of [15], [1], [22] and [21] are provided. From this table one can see the efficiency and applicability of the suggested method for solving nonlinear quasi bang-bang OCPs.

\section{CONCLUSIONS}

In this paper, an efficient pseudospectral approach has been generalizied for obtaining the numerical solution of nonlinear quasi bang-bang OCPs. The present 
AN EFFICIENT LEGENDRE PSEUDOSPECTRAL METHOD

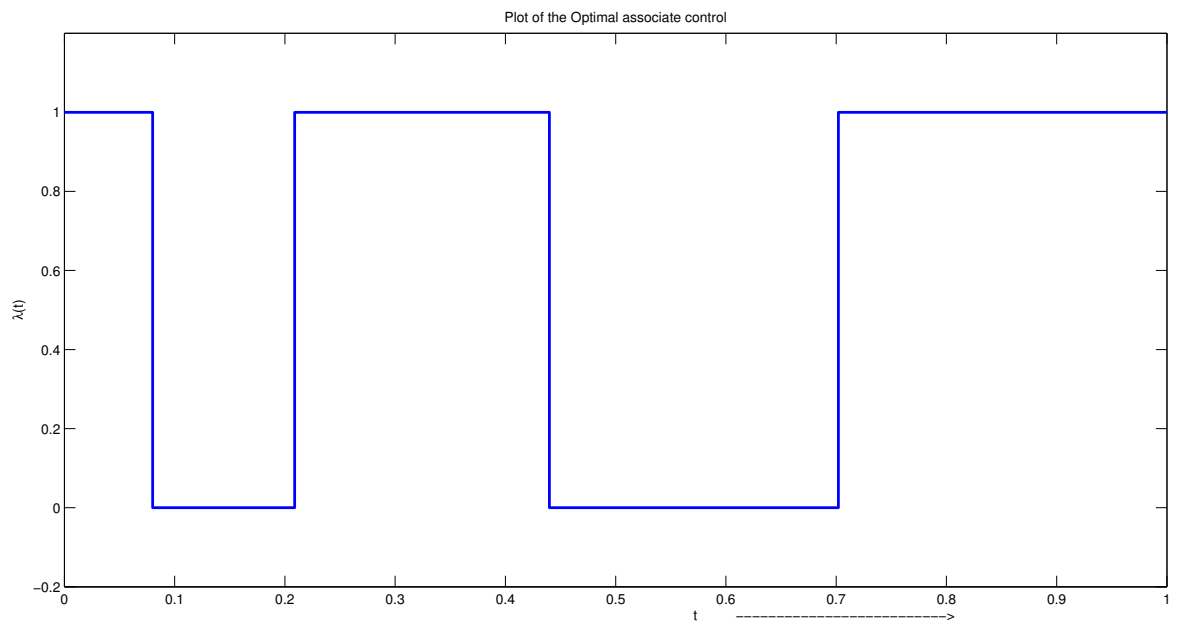

Fig. 2. Numerical solution of the optimal associated control $\lambda(t)$ for $N=16$

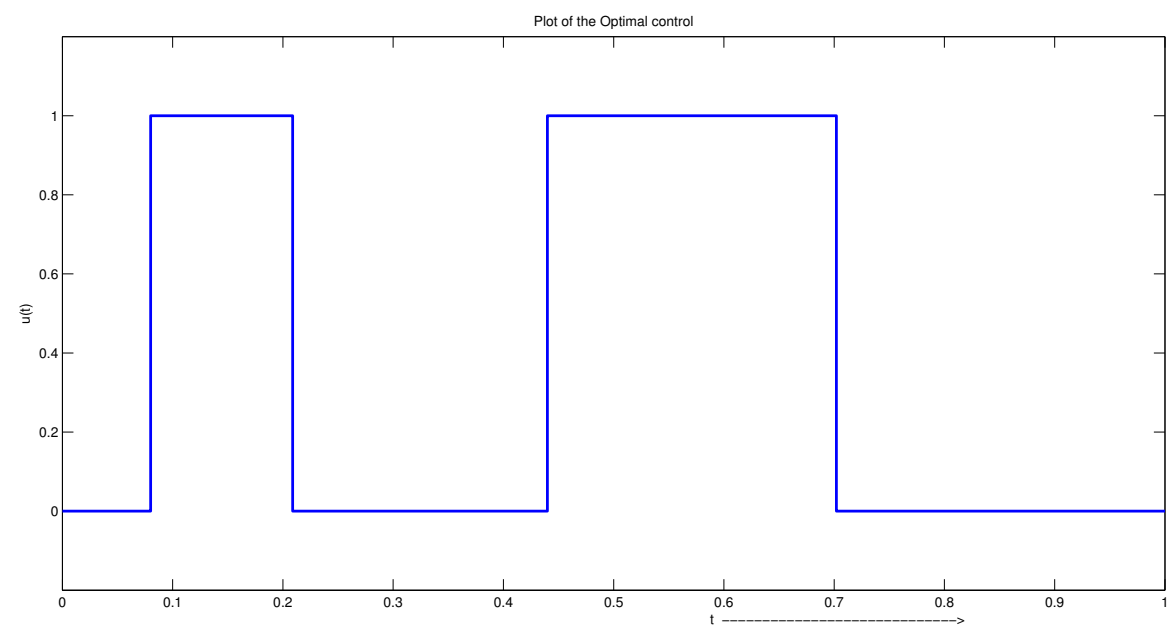

Fig. 3. Numerical solution of the optimal control $u(t)$ for $N=16$

method use the general idea of [20] for dealing with such problems. As illustrated in the numerical implementation, the proposed scheme has superior results with respect to at least 4 methods which confirm the efficiency of the presented method. The suggested approach is applicable and provides very accurate results even by using a small number of collocation points. According to the numerical experiments, the presented method is more sensitive to initial guess in comparison with the traditional pseudospectral methods. However the presented method is terminated successfully by providing an initial guess from the solution of the classical 
EMRAN TOHIDI, SOMAYYEH LOTFI

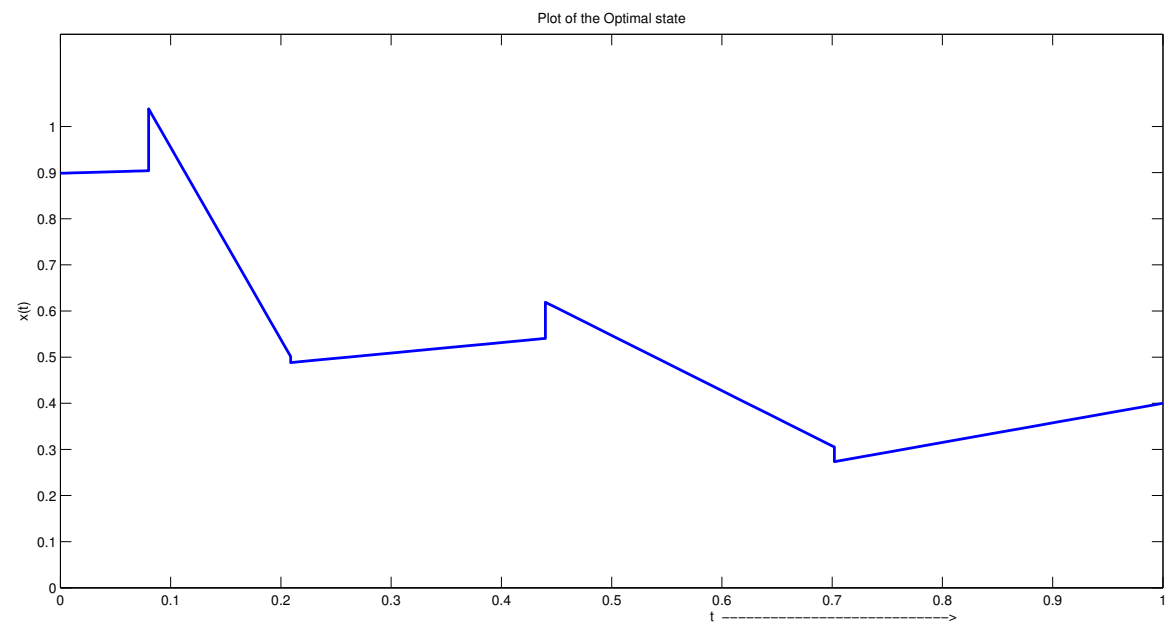

Fig. 4. Numerical solution of the optimal state $x(t)$ for $N=16$

pseudospectral methods, even with small $N$.

\section{REFERENCES}

[1] K. P. Badakhshan and A. V. Kamyad, Numerical Solution of Nonlinear Optimal Control Problems Using Non-linear Programming, Appl. Math. Comput, 187 (2007), 1511-1519.

[2] R. E. Bellman, Dynamic Programming, Princeton University Press, Princeton, NJ, 1957.

[3] J. T. Betts, Survey of numerical methods for trajectory optimization. Journal of Guidance, Control, and Dynamics, 21 (1998) 193-207.

[4] J. T. Betts, Practical methods for optimal control using nonlinear programming, Advances in Design and Control 3, SIAM, Philadelphia, PA, 2001.

[5] A. E. Bryson, and Y. C. Ho, Applied optimal Control, Hemisphere, New York, 1975.

[6] C. Canuto, M. Hussaini, A. Quarteroni, T. A. Zang, Spectral Methods in Fluid Dynamics. Springer Series in Computational Physics. Springer: Berlin, 1991.

[7] M. Dehghan, M. Shamsi, Numerical solution of two-dimensional parabolic equation subject to nonstandard boundary specifications using the pseudospectral Legendre method. Numerical Methods for Partial Differential Equations, 22(2006), 1255-266.

[8] G. Elnagar, M. A. Kazemi, M. Razzaghi, The pseudospectral Legendre method for discretizing optimal control problems. IEEE Transactions on Automatic Control, 40 (1995), 1793-1796.

[9] F. Fahroo, I. M. Ross, Direct trajectory optimization by a Chebyshev pseudospectral method, J. Guidance. Control. Dynamics, 25(2002) 160-166.

[10] R. Fletcher, Practical Methods of Optimization, Wiley, Chichester, 1987.

[11] B. Fornberg, A Practical Guide to Pseudospectral Methods. Cambridge University Press: Cambridge, 1998.

[12] W. Kang, Q. Gong, I. M. Ross, Convergence of pseudospectral methods for nonlinear optimal control problems with discontinuous controller, 44th IEEE Conference on Decision and Control and European Control Conference (CDC-ECC'05), Sevil, Spain, 2005, 2799-2804.

[13] H. Ma, T. Qin, and W. zhang, An efficient Chebyshev algorithm for the solution of optimal control problems, IEEE trans. Autom. Control, 56 (2011) 675-680.

[14] J. Nocedal, S. J. Wright, Numerical Optimization, Springer Series in Operations Research. Springer, New York, NY, 1999. 


\section{AN EFFICIENT LEGENDRE PSEUDOSPECTRAL METHOD}

[15] M. H. Noori Skandari, E. Tohidi, Numerical solution of a class of nonlinear optimal control problems using linearization and discretization, Applied Mathematics, 2 (2011), 271-277.

[16] M, Razzaghi, G. Elnagar, Numerical solution of the controlled Duffing oscillator by the pseudospectral method, J. Comput. Appl. Math, 56 (1994) 253-261.

[17] M, Razzaghi, S. Yousefi, Legendre wavelets method for constrained optimal control problems, Math. Meth. Appl. Sci, 25 (2002), 529-539.

[18] I. M. Ross, J. Rea, F. Fahroo, Exploiting higher-order derivatives in computational optimal control. Proceedings of the 2002 IEEE Mediterranean Conference, Lisbon, Portugal, July 2002.

[19] M. Shamsi, M. Dehghan, Recovering a time-dependent coefficient in a parabolic equation from overspecified boundary data using the pseudospectral Legendre method, Numerical Methods for Partial Differential Equations, 23 (2007), 196-210.

[20] M. Shamsi, A modified pseudospectral scheme for accurate solution of Bang-Bang optimal control problems, Optimal, Control, Appl, Methods, DOI: 10.1002/oca.967.

[21] O. V. Stryk, Numerical Solution of Optimal Control Problems by Direct Collocation, In: R. Bulrisch, A. Miele, J. Stoer and K. H. Well, Eds., Optional Control of Variations, Optimal Control Theory and Numerical Methods, International Series of Numerical Mathematics, Birkhuser Verlag, Basel, 1993, 129-143.

[22] E. Tohidi, O. R. N. Samadi, M. H. Farahi, Legendre approximation for solving a class of nonlinear optimal control problems, Journal of Mathematical Finance, 1 (2011), 8-13.

[23] E. Tohidi, M. H. Noori Skandari, A New Approach for a Class of Nonlinear Optimal Control Problems Using Linear Combination Property of Intervals, Journal of Computations and Modelling, 1 (2011), 145-156.

[24] E. Tohidi, O. R. N. Samadi, Optimal control of nonlinear Volterra integral equations via Legendre Polynomials, IMA Journal of Mathematical Control and Information, DOI: 10.1093/imamci/DNS014.

[25] L. N. Trefethen, Spectral methods in Matlab. Software-Environments-Tools 10. IAM, Society for Industrial and Applied Mathematics: Philadelphia, PA, 2000.

[26] V. Yen, M. Nagurka, Linear quadratic optimal control via Fourier-based state parameterization. Journal of Dynamic Systems, Measurement and Control, 113 (1991), 206-215.

\section{EMRAN TOHIDI}

Department of Mathematics, Islamic Azad University,

Zahedan Branch, Zahedan, Iran

\section{SOMAYYEH LOTFI NOGHABI}

Department of Applied Mathematics, School of Mathematical Sciences, Ferdowsi University of Mashhad, Mashhad, Iran

Corresponding author: EMRAN TOHIDI

Email: emrantohidi@gmail.com

Received August 2012 